\title{
QUALIDADE DE VIDA RELACIONADA À VOZ DE PROFESSORES UNIVERSITÁRIOS
}

\section{Voice-related quality of life of university teachers}

\author{
Mariana Zerbetto Fabrício ${ }^{(1)}$, Silvia Tieko Kasama ${ }^{(2)}$, Edson Zangiacomi Martinez ${ }^{(3)}$
}

\begin{abstract}
RESUMO
Objetivo: observar a qualidade de vida relacionada à voz dos docentes da Faculdade de Medicina de Ribeirão Preto da Universidade de São Paulo (FMRP-USP), além de descrever seus conhecimentos referentes à saúde vocal, fornecendo assim, dados que possam motivar futuras ações para prevenção e tratamento da disfonia. Métodos: foram utilizados o Protocolo de Qualidade de Vida em Voz (QVV) e um questionário que investigou sintomas vocais, uso da voz e atitudes diante dos sintomas. Resultados: registrou-se que $82 \%$ dos docentes participantes atingiram escores iguais a 100 para o domínio sócio-emocional, 38\% para o domínio físico e 38\% para o escore total. Uma grande parte (31\%) considera sua voz "muito boa", mas deve-se considerar que $26 \%$ dos entrevistados não avaliaram sua voz. Com relação às atitudes que os docentes tomam frente a um problema vocal, $53 \%$ relatam diminuir o uso da voz. Os sintomas de maior incidência relatados pelos docentes foram garganta seca, tosse e perda de voz. Conclusão: o presente estudo mostrou relatos de satisfação vocal e alto índice de qualidade de vida, porém observou-se uma prevalência elevada de sintomas vocais, demonstrando a necessidade de ações preventivas e de orientação vocal para esses professores.
\end{abstract}

DESCRITORES: Voz; Docentes; Qualidade de Vida; Saúde do Trabalhador

\section{INTRODUÇÃO}

A voz é o som produzido por meio da vibração das pregas vocais e conta com a participação de várias estruturas que compõem o trato vocal, começando na laringe e terminando na cavidade oral e/ ou nariz. É por meio dela, um componente importante na comunicação interpessoal, que o homem se expressa. " $A$ voz é uma das extensões mais fortes da nossa personalidade, nosso sentido de interrelação na comunicação interpessoal, um meio

(1) Aluna do curso de Graduação em Fonoaudiologia da Faculdade de Medicina de Ribeirão Preto da Universidade de São Paulo, FMRP-USP, Ribeirão Preto, SP, Brasil; Bolsista de Iniciação Científica do Programa Institucional de Bolsas de Iniciação Científica do Conselho Nacional de Desenvolvimento Científico e Tecnológico, PIBIC - CNPq.

(2) Fonoaudióloga; Pós-graduanda do Programa de Pósgraduação em Saúde na Comunidade da Faculdade de Medicina de Ribeirão Preto da Universidade de São Paulo, FMRP-USP, Ribeirão Preto, SP.

(3) Estatístico; Professor Livre Docente do Departamento de Medicina Social da Faculdade de Medicina de Ribeirão Preto da Universidade de São Paulo, FMRP-USP, Ribeirão Preto, SP.

Conflito de interesses: inexistente essencial de atingir o outro" ${ }^{~}$. Ela enriquece a transmissão da palavra, mensagem ou sentimento, tanto socialmente quanto profissionalmente. Portanto, é esperado que os profissionais que utilizam a voz como instrumento de trabalho tenham uma voz bem projetada, com articulação precisa, coordenação pneumofonoarticulatória, boa sonoridade, ritmo e velocidades adequados, mostrando assim, clareza de ideias. "A voz é um dos principais instrumentos de trabalho dos professores e estará cumprindo seu papel quanto melhor projetada e mais adequada sua intensidade" ${ }^{2}$. Porém, faltam a estes profissionais informações a respeito da produção vocal, além de orientações sobre o próprio uso e cuidados para preservá-la, o que poderia evitar abusos, posturas e hábitos inadequados. Estudos evidenciam que a falta de conhecimento acerca da própria $\mathrm{voz}^{3,4} \mathrm{e}$ a dificuldade de autopercepção, por esta ser uma medida subjetiva, são fatos comuns entre os professores. Tensões na musculatura cervical, postura inadequada, falar sem parar por horas seguidas, padrão respiratório inadequado, alterando tom, agudizando repentinamente no momento do grito, voz abafada, presa, sem projeção são características frequentemente encontradas entre os professores ${ }^{3}$. 
Os professores apresentam alto risco para desenvolver o distúrbio vocal de ordem ocupacional ${ }^{5-8}$. Um estudo com professores da rede pública de Mogi das Cruzes mostrou que $80,7 \%$ destes profissionais referiram algum grau de disfonia ${ }^{9}$. Já em Ribeirão Preto, a prevalência de queixa da disfonia entre os professores da rede pública municipal foi estimada em outro estudo em $93 \%{ }^{10}$. E ainda, dos 734 professores da prefeitura do município de São Paulo que participaram de uma pesquisa ${ }^{4}, 60 \%$ fazem referência (atual ou pregressa) de alteração vocal. As relações sociais no trabalho e sua vida particular ajudam a deixar esse profissional mais susceptível ao estresse, contribuindo para as condições de mau uso e abuso vocal. Muitas vezes os professores universitários são considerados de elite, pelas condições mais favoráveis de trabalho quando comparados aos professores de outros níveis de ensino. Entretanto, sua jornada de trabalho é significativa ${ }^{11}$, requerendo alta demanda vocal. Além disso, o estresse, a competitividade e a responsabilidade são intensos, fatores estes que podem predispor o professor a problemas de voz.

Em um inquérito com 489 professores da Universidade de Fortaleza ${ }^{12}$, verificou-se uma prevalência média de problemas de voz de 20,2 para cada 100 professores. Os sintomas vocais mais referidos após uma jornada em sala de aula foram ressecamento, pigarro, ardor e rouquidão. Apesar dos professores referirem rouquidão com frequência, apenas $6,6 \%$ relatam buscar auxílio médico ou fonoaudiológico. O uso de métodos paliativos (balas de hortelã, gargarejos, automedicação) superou as condutas realmente eficazes para a melhoria dos problemas de voz.

A qualidade de vida relacionada à voz é um importante auxílio para o entendimento da percepção que as pessoas possuem em relação à sua saúde vocal e de suas reações perante as alterações na voz. O protocolo QVV ${ }^{13}$ (Qualidade de Vida e Voz) é um instrumento desenvolvido para medir a relação da voz com a qualidade de vida, o qual já foi traduzido, adaptado ${ }^{14} \mathrm{e}$ validado ${ }^{15}$ para a língua portuguesa. Trata-se de um protocolo com 10 itens, considerando três domínios: sócio-emocional, físico e global.

Tendo os problemas de voz uma relação tão estreita com as condições de trabalho do professor, estas precisam ser elucidadas e melhor compreendidas. Assim, o presente estudo tem por objetivo avaliar a qualidade de vida relacionada à voz dos docentes da Faculdade de Medicina de Ribeirão Preto (FMRP) da Universidade de São Paulo (USP), por meio de um instrumento estruturado, e verificar as suas atitudes mais frequentes em relação à saúde vocal, fornecendo dados que possam motivar futuras ações que permitam auxiliá-los na prevenção e tratamento da disfonia.

\section{MÉTODOS}

Foi realizada uma pesquisa de caráter observacional e de corte transversal na Faculdade de Medicina de Ribeirão Preto (FMRP) da Universidade de São Paulo (USP). Foram convidados a participar da pesquisa todos os 306 docentes da FMRP, então distribuídos em 13 Departamentos, sem especificar critérios de inclusão ou exclusão. Esta casuística é composta de profissionais de ambos os sexos, sem restrições quanto à idade. Foram enviadas em anexo ao instrumento de coleta de dados uma carta de informação, explicando os objetivos e procedimentos da pesquisa, sendo solicitado aos participantes a assinatura de um termo de consentimento, autorizando a utilização dos dados obtidos.

O instrumento de coleta de dados utilizado foi o protocolo de Qualidade de Vida em Voz (QVV) ${ }^{13-16}$, adaptado do inventário norte-americano Voice Related Quality of Life (V-RQOL) ${ }^{13}$. O instrumento analisa o impacto da disfonia na qualidade de vida do sujeito segundo 10 itens em três domínios: sócio-emocional (questões 4, 5, 8 e 10), físico (questões 1, 2, 3, 6, 7 e 9) e global (todas as questões). Estes domínios apresentam valores que, depois de padronizados, variam entre zero e cem, sendo considerados piores os valores mais próximos de zero e melhores os mais próximos de cem. Além das variáveis contidas no QVV, foram coletados os seguintes dados: tempo de trabalho como professor, número médio de aulas proferidas por semana naquele semestre, número de alunos por sala de aulas, percepção do ambiente acústico da sala; queixas de alterações vocais; auto-avaliação vocal, realização de atividades fora do âmbito escolar que exigem o uso da voz, e histórico de passagem por consulta fonoaudiológica.

Depois de preenchido, o instrumento e uma cópia do Termo de Consentimento foram enviados pelo respondente para a Secretaria do Departamento de Medicina Social da FMRP. O trabalho, de acordo com seus aspectos éticos, garantiu ao docente sigilo e privacidade. O projeto e o respectivo Termo de Consentimento Livre e Esclarecido (TCLE) foram aprovados pelo Comitê de Ética em Pesquisa do Hospital das Clínicas da FMRP da USP, de acordo com o Processo HCRP no 4545/2007.

A tabulação dos dados foi feita no programa SAS, sendo utilizadas apenas estatísticas descritivas. 


\section{RESULTADOS}

Responderam ao questionário 82 docentes, sendo a taxa de retorno de $30,4 \%$. Um docente foi excluído da análise, dado que ele não respondeu aos itens do QVV. Dentre os respondentes, 44 (54\%) são do sexo masculino e 37 (46\%) do sexo feminino. A média do tempo de docência dos participantes foi de 25 anos. O número médio de aulas ministradas por semana foi de 11,7 horas, sendo a média de alunos por sala de aula de 33 .

A Tabela 1 mostra uma descrição dos escores do QVV, segundo os domínios sócio-emocional, físico e total.

A Figura 1 ilustra a distribuição das respostas a cada um dos dez itens do QVV, em frequências absolutas. O protocolo QVV solicita ainda ao respondente que avalie a sua voz em uma escala de 5 pontos, de "excelente" a "ruim". Metade dos professores avaliou a sua voz como excelente ou muito boa, enquanto apenas $3 \%$ a avaliaram como ruim, como mostra a Figura 2.

A Tabela 2 apresenta os sintomas relatados pelos docentes como presentes em seu cotidiano profissional. As respostas das questões relacionadas ao uso da voz podem ser observadas na Tabela 3.

Quanto às atitudes dos professores diante do problema vocal, $53 \%$ relatam diminuir o uso da voz, $30 \%$ relatam não fazer nada, e $21 \%$ usam alguma solução caseira, como visualizado na Figura 3.

\section{DISCUSSÃO}

Nesta pesquisa, nota-se que $82 \%$ dos docentes participantes atingiram escores iguais a 100 para o domínio sócio-emocional. A literatura considera que valores de QVV iguais a 50 podem ser considerados como impacto moderado da voz no domínio respectivo ${ }^{17}$. Visto que a maioria dos docentes obteve escores de QVV maiores que cinquenta em todos os três domínios (Tabela 1), com medianas relativamente altas, pode-se considerar que os docentes que participaram da pesquisa apresentam uma boa qualidade de vida relacionada à voz, segundo a avaliação do QVV.

A auto-avaliação vocal dos participantes desse estudo pode ser considerada positiva, pois a maioria considerou sua voz "muito boa" ou "boa" (Figura 2). Este dado condiz com dados de outros estudos ${ }^{13,16}$, nos quais para bons escores de QVV, já se espera boa auto-avaliação vocal.

Na Figura 1, observa-se que dentre os itens do domínio sócio-emocional (questões 4, 5, 8 e 10), o item 4 (associado ao sentir-se ansioso ou frustrado pela própria voz) é o que recebeu um menor número de respostas diferentes do rótulo "1" ("não é um problema"), o que mostra que a voz não traz aos docentes sentimentos de depressão ou motivos para evitarem o contato social ou tornarem-se expansivos em suas relações. Por outro lado, é relativamente grande o número de docentes (51\%) que apontaram alguma dificuldade para serem ouvidos em lugares barulhentos (questão 1 do QVV, onde $49 \%$ afirmaram não ter este problema). A docência geralmente envolve o uso da voz em ambientes ruidosos ${ }^{17}$, o que leva o professor à elevação da intensidade vocal pela competição sonora e necessidade de superar o ruído ambiente. Além da sugestão de diminuir a competição sonora, o professor deveria ser orientado a respeito de exercícios para melhorar a resistência e a projeção vocal.

$\mathrm{Na}$ Tabela 3, observa-se que 55 (68\%) professores relataram algum incômodo por ruídos internos e/ou externos à sala de aula. O incômodo com ruídos está presente no cotidiano das diversas categorias de profissionais de ensino, sendo que em um estudo com professores de escolas municipais de São Paulo ${ }^{4}$, 65\% consideram o ambiente de trabalho ruidoso. Os achados do presente estudo corroboram outros autores ${ }^{18,19}$, que afirmam que na universidade a docência se realiza geralmente em condições nem sempre satisfatórias quanto à acústica, com presença de ruídos internos e externos à sala de aula, que interferem no desenvolvimento da aula.

Tabela 1 - Descrição dos escores do QVV, segundo os domínios sócio-emocional, físico e total

\begin{tabular}{lccccccc}
\hline Domínio & $\boldsymbol{n}$ & mínimo & 1quartil & mediana & 3 quartil & máximo & $\begin{array}{c}\text { Respondentes } \\
\text { com escores } \\
\text { iguais a 100 }\end{array}$ \\
\hline Sócioemocional & 81 & 50,0 & 100,0 & 100,0 & 100,0 & 100,0 & $82 \%$ \\
Físico & 81 & 50,0 & 87,5 & 95,8 & 100,0 & 100,0 & $38 \%$ \\
Global & 81 & 52,5 & 92,5 & 97,5 & 100,0 & 100,0 & $38 \%$ \\
\hline
\end{tabular}




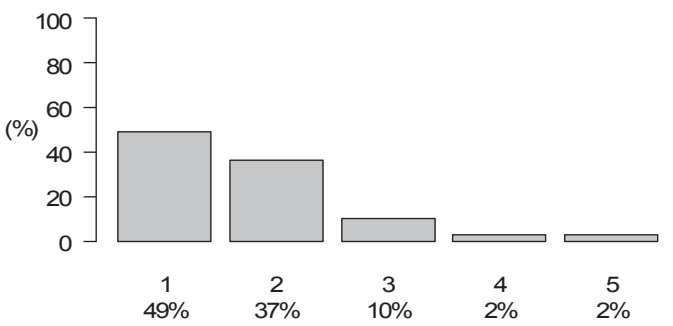

1. Tenho dificuldades em falar forte (alto) ou ser ouvido em lugares barulhentos.

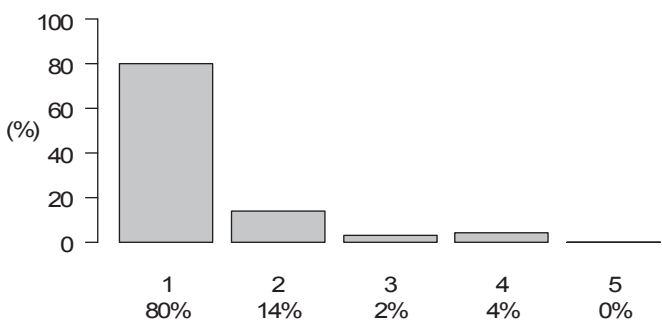

3. Às vezes, quando começo a falar não sei como minha voz vai sair.

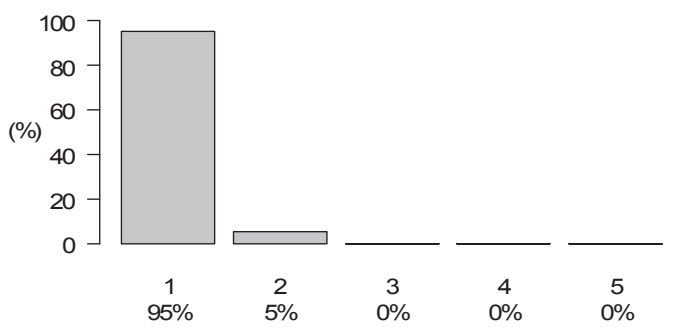

5. Às vezes, fico deprimido (por causa da minha voz).

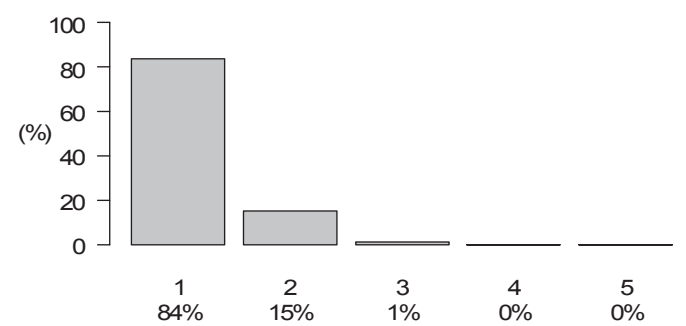

7. Tenho problemas no meu trabalho ou para desenvolver minha profissão (por causa da minha voz).

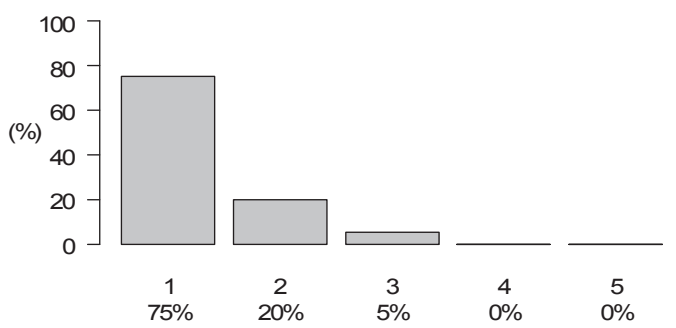

9. Tenho que repetir o que falo para ser compreendido.

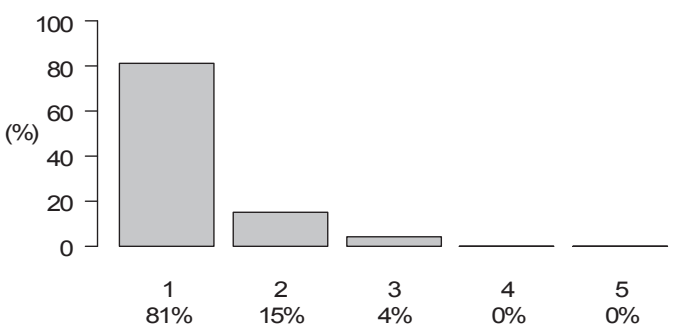

2. O ar acaba rápido e preciso respirar muitas vezes enquanto eu falo.

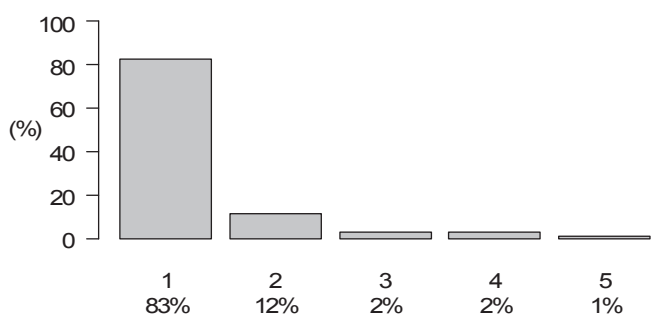

4. Às vezes, fico ansioso ou frustrado (por causa da minha voz).

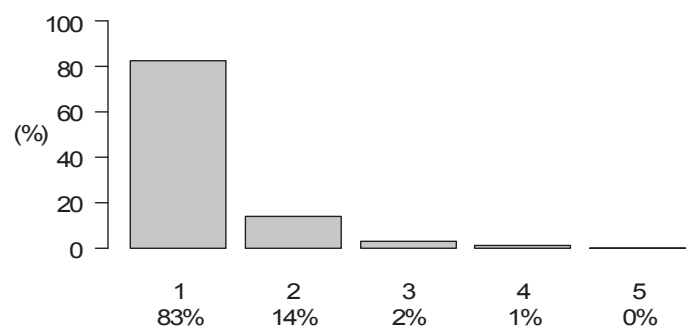

6. Tenho dificuldades ao telefone (por causa da minha voz).

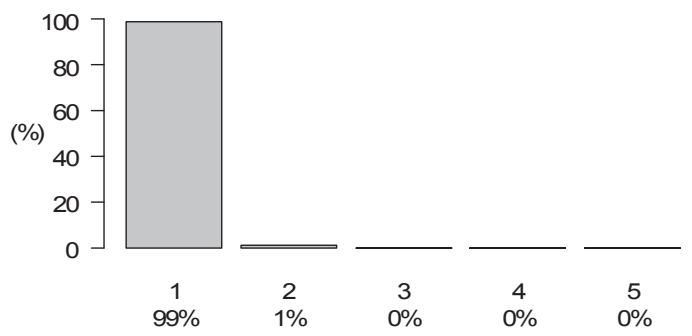

8. Evito sair socialmente (por causa da minha voz).

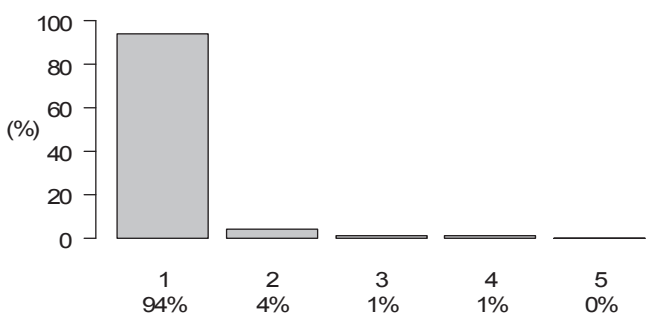

10. Tenho me tornado menos expansivo (por causa da minha voz). 


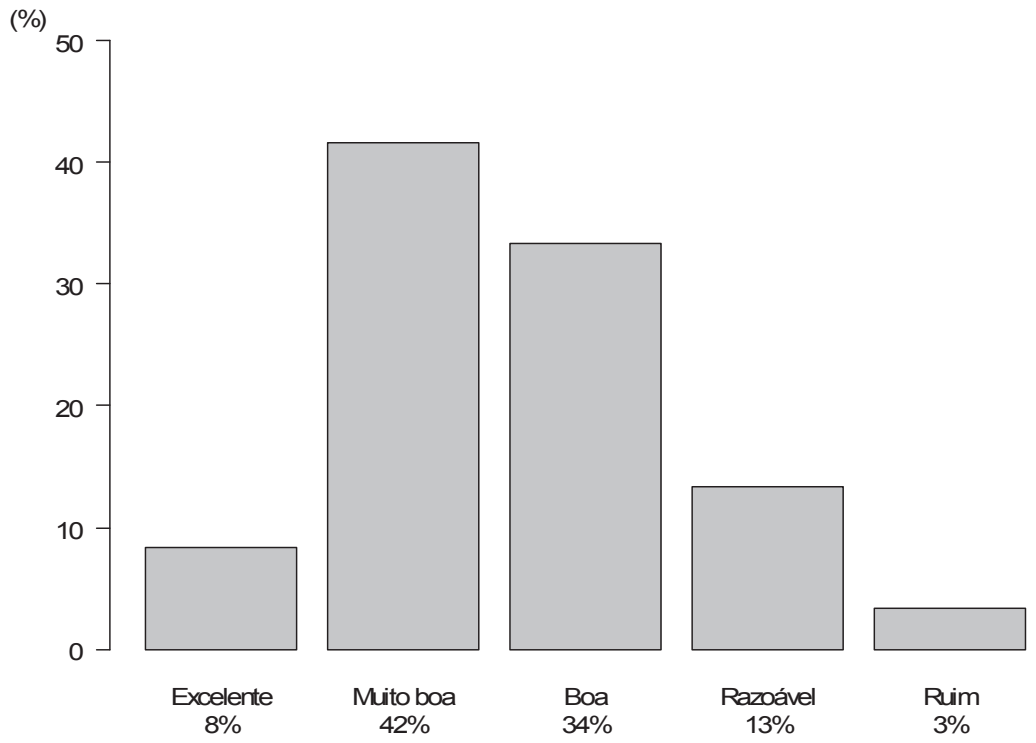

Figura 2 - Auto-avaliação vocal dos professores (\%)

Tabela 2 - Frequência referida de sintomas vocais

\begin{tabular}{lcccc}
\hline & \multicolumn{4}{c}{ Frequência referida } \\
\cline { 2 - 4 } Sintoma & sempre & às vezes & nunca & não opinou \\
\hline Rouquidão & $2(2 \%)$ & $41(51 \%)$ & $35(43 \%)$ & $3(8 \%)$ \\
Pigarro & $3(4 \%)$ & $38(47 \%)$ & $33(41 \%)$ & $6(8 \%)$ \\
Tosse & $1(1 \%)$ & $53(66 \%)$ & $21(26 \%)$ & $5(7 \%)$ \\
Desconforto ao falar & $1(1 \%)$ & $20(25 \%)$ & $52(65 \%)$ & $7(9 \%)$ \\
Garganta seca & $5(6 \%)$ & $48(60 \%)$ & $22(28 \%)$ & $5(6 \%)$ \\
Perda de voz & $0(0 \%)$ & $18(23 \%)$ & $57(71 \%)$ & $5(6 \%)$ \\
Dor na garganta & $0(0 \%)$ & $39(48 \%)$ & $38(46 \%)$ & $4(6 \%)$ \\
Variações de voz ao longo do dia & $0(0 \%)$ & $26(32 \%)$ & $49(61 \%)$ & $6(7 \%)$ \\
\hline
\end{tabular}

Tabela 3 - Questões relacionadas ao uso da voz

\begin{tabular}{lccc}
\hline \multirow{2}{*}{ Questões } & \multicolumn{3}{c}{ Frequências } \\
\cline { 2 - 4 } & Sim & Não & $\begin{array}{c}\text { Não } \\
\text { respondeu }\end{array}$ \\
\hline Incômodo por ruídos internos e/ou externos à sala de aula & $55(68 \%)$ & $24(30 \%)$ & $2(2 \%)$ \\
Necessidade de usar a voz mais alta que a habitual & $37(46 \%)$ & $43(53 \%)$ & $1(1 \%)$ \\
Problema vocal & $47(58 \%)$ & $34(42 \%)$ & 0 \\
Afastamento do trabalho & $3(4 \%)$ & $78(96 \%)$ & 0 \\
Consulta fonoaudiológica & $4(5 \%)$ & $76(94 \%)$ & $1(1 \%)$ \\
Hábitos como cigarro e/ou álcool & $17(21 \%)$ & $64(79 \%)$ & 0 \\
Atividade que utilize a voz fora do ambiente escolar & $41(51 \%)$ & $40(49 \%)$ & 0 \\
Conhecimento sobre o uso da voz & $37(46 \%)$ & $44(54 \%)$ & 0 \\
\hline
\end{tabular}




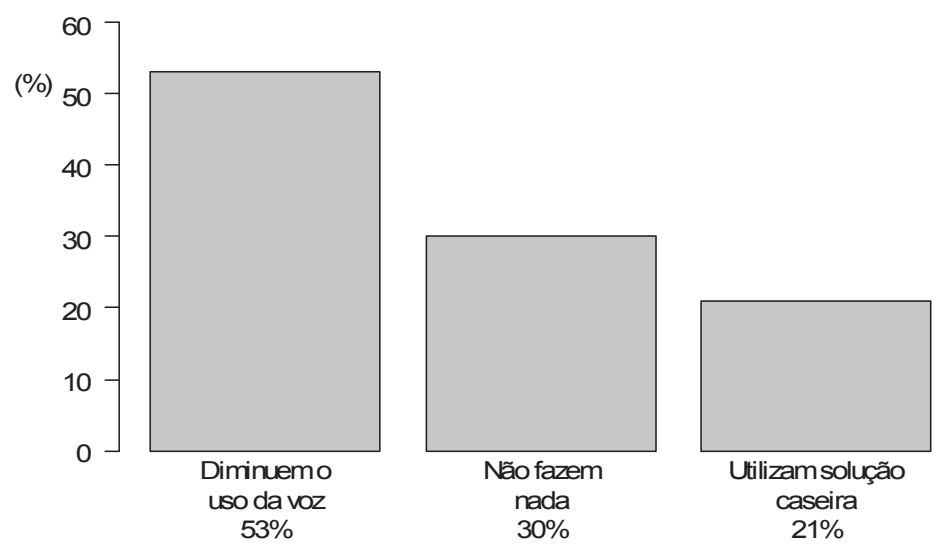

Figura 3 - Atitudes diante do problema vocal (\%)

Ainda na Tabela 3, nota-se que o número de docentes que ficaram afastados por algum problema vocal é pequeno (4\%) se comparado a um estudo com profissionais que utilizam a voz como instrumento de trabalho ${ }^{20}$, incluindo professores, no qual o exercício laboral foi a causa de $40 \%$ dos afastamentos. Em outro estudo ${ }^{21}$, dos 72 sujeitos com queixas vocais, 14 informaram que obtiveram afastamento médico em decorrência de problemas de voz. Em outra pesquisa ${ }^{19}, 15 \%$ dos docentes pesquisados já faltaram ao trabalho devido a distúrbios vocais. Além disso, o número de docentes que passou por uma consulta fonoaudiológica em algum momento é baixo diante da elevada porcentagem de docentes que referiram ter ou já ter tido alteração vocal (58\%). Por outro lado, deve-se considerar que a presente pesquisa foi realizada em uma faculdade cujos respondentes são formados na área da saúde, inclusive em Fonoaudiologia e supõe-se que não necessariamente procurem atendimento especializado por já terem conhecimento na área.

Ainda que os docentes da FMRP apresentem um grau de satisfação vocal elevado, é consenso ${ }^{12}$ que a auto-avaliação vocal merece algumas reflexões, pois dentre os profissionais da voz falada, a categoria docente é a que apresenta maior prevalência de alterações vocais e disfonias. Além disso, é visto que há ocorrência de vozes alteradas e dificuldades ao falar em professores que as avaliam favoravelmente ${ }^{17}$. Mesmo tratando-se de profissionais da saúde, a hipótese destes docentes não conseguirem identificar os problemas ou não darem devida importância aos sinais e sintomas de uma alteração vocal é pertinente. Se um indivíduo desconhece sua própria voz, pode não perceber a perturbação de suas qualidades, assim como não associa os sintomas vocais ao uso incorreto da voz. Como a deteriorização da qualidade da voz é progressiva, o indivíduo vai gradativamente assumindo como normal ou correto o padrão vocal desviado e identificando-se com ele. A literatura evidencia que os professores têm apenas um conhecimento básico do mecanismo necessário à produção da voz ${ }^{19}$, estando este ainda restrito ao aspecto orgânico com referência às pregas vocais ou à necessidade de ar para a produção do som.

No presente estudo, por tratar-se de profissionais de saúde e apesar de alguns participantes terem afirmado possuir conhecimento sobre o uso da voz, vale destacar as atitudes tomadas diante do problema vocal: diminuir o uso da voz, não fazer nada e utilizar soluções caseiras (Figura 3) são atitudes que não resolverão a causa de uma possível disfonia. Estes dados demonstram que falta orientação para os professores sobre como identificar e como lidar com o problema vocal.

Nota-se que muitos docentes relatam que às vezes apresentam rouquidão, pigarro, tosse ou garganta seca (Tabela 2), dado semelhante ao encontrado entre os professores da Universidade de Fortaleza ${ }^{12}$, cujos sintomas vocais mais referidos após uma jornada em sala de aula foram ressecamento, pigarro, ardor e rouquidão.

Ao encontrar uma alta prevalência de distúrbios de voz em um estudo ${ }^{12}$ que envolveu professores universitários $(20,2 \%)$, seus autores propuseram dentre outras estratégias, a oferta sistemática de cursos de aperfeiçoamento vocal aos professores e sondagem acerca dos problemas de voz a cada 2 anos para verificar a eficácia das medidas preventivas. Estudos recentes ${ }^{22,23}$ mostram que programas de prevenção são importantes e devem focar na conscientização dos problemas pelos profissionais, reconhecimento dos sintomas precoces (fadiga vocal, tosse seca, e alteração do pitch vocal), mas também na orientação sobre uma boa higiene vocal. Diante destes resultados, e dos achados do 
presente estudo, vê-se necessário uma maior preocupação em relação à prevenção primária junto a esses professores.

\section{CONCLUSÃO}

A maioria dos docentes que participaram da pesquisa apresenta uma boa qualidade de vida associada à voz, segundo a avaliação do QVV, mas foi evidenciada uma prevalência elevada de sintomas vocais. Tal dado demonstra a necessidade de ações preventivas e de orientação vocal para esses professores.

\section{AGRADECIMENTOS}

Ao Conselho Nacional de Desenvolvimento Científico e Tecnológico (CNPq) pelo apoio financeiro.

\begin{abstract}
Purpose: to observe the voice-related quality of life of teachers from the Ribeirão Preto Medical School, University of São Paulo (FMRP/USP), to describe their knowledge on vocal health, and to provide data that can motivate future actions for preventing and treating dysphonia. Methods: data collection instrument was Quality of Life and Voice Protocol (QLV). It was also used a questionnaire that investigated vocal symptoms, vocal use and attitudes before the symptoms. Results: $82 \%$ of the teachers who participated in the survey achieved scores equal to 100 for the socio-emotional domain, $38 \%$ for the physical domain and $38 \%$ for the total score. A large portion $(31 \%)$ believe his voice "very good" but it must be considered that $26 \%$ of respondents did not examine their voice. Regarding the question about the attitudes that teachers take before a vocal problem, $53 \%$ reported decrease in the use of voice. The higher incidence of symptoms reported by teachers was dry throat, cough and voice loss. Conclusion: this study showed reports of voice satisfaction and high quality of life, but there was a high prevalence of vocal symptoms, demonstrating the need for preventive actions and voice education for these university teachers.
\end{abstract}

KEYWORDS: Voice; Faculty; Quality of Life; Occupational Health

\section{REFERÊNCIAS}

1. Behlau M, Pontes P. Avaliação e tratamento das disfonias. São Paulo: Lovise; 1995.

2. Assis JR. Avaliação do conhecimento relativo à saúde vocal em acadêmicos do curso normal superior de uma universidade. [dissertação] Franca (SP): Universidade de Franca; 2005.

3. Carelli EG, Nakao M. Educação vocal na formação do docente. Fono Atual. 2002; 5(22):40-52.

4. Ferreira LP, Giannini SPP, Figueira S, Silva EE, Karmann DF, Thomé de Souza TM. Condições de produção vocal de professores da Prefeitura do Município de São Paulo. Dist Comun. 2003; 14(2):275-307.

5. Medeiros AM, Barreto SM, Assunção AA. Voice disorders (dysphonia) in public school female teachers working in Belo Horizonte: prevalence and associated factors. J Voice. 2008; 22(6):676-87.

6. Munier C, Kinsella R. The prevalence and impact of voice problems in primary school teachers. Occup Med. 2008; 58(1):74-6.
7. Sliwinska-Kowalska M, Niebudek-Bogusz E, Fiszer M, Los-Spychalska T, Kotylo P, SznurowskaPrzygocka B, et al. The prevalence and risk factors for occupational voice disorders in teachers. Folia Phoniatr Logop. 2006; 58(2):85-101.

8. Araújo TM, Reis EJFB, Carvalho FM, Porto LA, Reis IC, Andrade JM. Fatores associados a alterações vocais em professores. Cad Saúde Pública. 2008; 24(6):1229-38.

9. Fuess VLR, Lorenz MC. Disfonia em professores do ensino municipal: prevalência e fatores de risco. Rev Bras Otorrinolaringol. 2003; 69(6):807-12.

10. Defina-lqueda AP. Auto-percepção da voz e interferências de problemas vocais: um estudo com professores da rede municipal de Ribeirão Preto/ SP. [dissertação], Ribeirão Preto (SP): Universidade de São Paulo; 2006.

11. Polizzi JA, Barría MA, Campos A. Disfonía funcional y evaluación fonoaudiológica de un grupo de docentes universitarios. Rev Otorrinolaringol Cir Cabeza Cuello. 1986; 46(2):81-4.12. Pordeus AMJ, Palmeira CT, Pinto VCV. Inquérito de prevalência de 
problemas da voz em professores da Universidade de Fortaleza. Pró-Fono. 1996; 8(2):15-24.

13. Hogikyan ND, Sethuraman G. Validation of an instrument to measure voice-related quality of life (V-RQOL). J Voice. 1999; 13(4):557-69.

14. Behlau M, Madazio G, Feijó D, Pontes P. Avaliação de voz. In: Behlau M. Voz: o livro do especialista. Vol 1. Rio de Janeiro: Revinter; 2001. p. $120-23$.

15. Gasparini GGO. Validação do questionário de Avaliação de Qualidade de Vida em Voz (QVV). [dissertação] São Paulo (SP): Universidade Federal de São Paulo; 2005.

16. Kasama ST, Brasolotto AG. Percepção vocal e qualidade de vida. Pró-Fono. 2007; 19(1):19-28.

17. Grillo MHMM, Penteado RZ. Impacto da voz na qualidade de vida de professore(a)s do ensino fundamental. Pró-Fono. 2005; 17(3):321-30.

18. Lemos S, Rumel D. Ocorrência de disfonia em professores de escolas públicas da rede municipal de ensino de Criciúma-SC. Rev Bras Saúde Ocup. 2005; 30(112):7-13.
19. Servilha EAM. Consciência vocal em docentes universitários. Pró-Fono. 1997; 9(2):53-61.

20. Ortiz E, Costa EA, Spina AL, Crespo AN. Proposta de modelo de atendimento multidisciplinar para disfonias relacionadas ao trabalho: estudo preliminar. Rev Bras Otorrinolaringol. 2004; 70(5):590-6.

21. Fabron EMG, Omote S. Queixas vocais entre professores e outros profissionais In: Ferreira LPC, Costa HO. Voz ativa: falando sobre o profissional da voz. São Paulo: Roca; 2000. p. 91-100.

22. Fortes FSG, Imamura R, Tsuji DH, Sennes LU. Perfil dos profissionais da voz com queixas vocais atendidos em um centro terciário de saúde. Rev Bras Otorrinolaringol. 2007; 73(1):27-31.

23. Niebudek-Bogusz E, Sznurowska-Przygocka B, Fiszer M, Kotyło P, Sinkiewicz A, Modrzewska $M$, et al. The effectiveness of voice therapy for teachers with dysphonia. Folia Phoniatr Logop. 2008; 60(3):134-41.

RECEBIDO EM: 13/12/2008

ACEITO EM: 11/09/2009

Endereço para correspondência:

Edson Zangiacomi Martinez

Depto. de Medicina Social, FMRP/USP

Av. Bandeirantes, 3900

Ribeirão Preto - SP

CEP: $14049-900$

E-mail: edson@fmrp.usp.br 